\title{
The Nocturnal Return of Neglected Regrets: Deficits in Regret Anticipa- tion Predict Insomnia Symptoms
}

\author{
Ralph E. Schmidt* and Martial Van der Linden
}

Swiss Center for Affective Sciences / University of Geneva, 7, rue des Battoirs / CH-1205 Geneva, Switzerland

\begin{abstract}
Cognitive accounts of insomnia generally assume that excessive mental activity at bedtime constitutes a central impediment to the process of falling asleep, yet the affective aspects of this mental hyperactivity still remain largely unexplored. The present study explored whether failures in regret anticipation might lead to a rise of regret-related counterfactual thoughts and emotions at bedtime, thereby hindering or interrupting sleep. A sample of 105 undergraduate students completed the newly developed Regret Anticipation Failures Scale (RAFS), the Bedtime Counterfactual Processing Questionnaire (BCPQ), and the Insomnia Severity Index (ISI). Analyses revealed that both regret anticipation failures and bedtime counterfactual processing were related to insomnia severity and that the effect of regret anticipation failures on insomnia was mediated by the frequency of regret-related thoughts and emotions at bedtime. These findings reveal for the first time that failures in antecedent-focused emotion regulation may contribute to sleeping difficulties.
\end{abstract}

Keywords: Affective forecasting, antecedent-focused emotion regulation, counterfactual thinking, insomnia, regret anticipation, rumination.

\section{INTRODUCTION}

Insomnia ranks among the most frequent health complaints. In a recent poll of the National Sleep Foundation, $64 \%$ of the respondents indicated that, within the past month, they had experienced at least one symptom of insomnia a few nights a week or more often, and $41 \%$ reported experiencing such symptoms every night or almost every night [1]. The individual and socioeconomic impact of insomnia is considerable: The consequences of poor sleep include impaired concentration and memory, heightened risk of accidents, increased risk of medical conditions such as cardiovascular disease, more frequent use of healthcare services, and augmented work absenteeism [2].

Over the past decades, cognitive approaches to insomnia have led to important advances in the modeling and treatment of this disorder [3, 4]. According to these approaches, excessive cognitive activity at bedtime may be considered as a central impediment to the process of falling asleep $[5,6]$. More recently, the importance of investigating affective processes in insomnia has been recognized [7] and a corresponding research agenda has been formulated [8]. The relevance of an affective approach to insomnia may be illustrated by a pioneering study revealing that gratitude, as assessed by the questionnaire GQ-6 [9], predicted shorter sleep onset latency, longer sleep duration as well as greater subjective sleep quality, and that the effect of gratitude on sleep was mediated by more positive and fewer negative pre-sleep cognitions [10].

Given that bedtime may often be the first quiet period in the course of the day available to review one's behavior, we

*Address correspondence to this author at the Swiss Center for Affective Sciences / University of Geneva, 7, rue des Battoirs / CH-1205 Geneva, Switzerland; Tel: 00412237998 20; Fax: 00412237998 01;

E-mail: Ralph.Schmidt@unige.ch have recently proposed that this time window might be particularly suitable for the emergence of feelings of regret, shame, and guilt, which, in turn, might interfere with the process of falling asleep [11]. Regret, shame, and guilt are generally conceptualized as counterfactual emotions because they involve comparing the facts of reality with counterfactual imaginations of what might have been (e.g., "If I had [not] done X, I would be in a better situation now"; e.g., [12]; see Appendix for terminology). The results of an exploratory study with university students supported our assumption [11]: Counterfactual thoughts and emotions were often experienced at bedtime and their frequency was linked to insomnia severity. Moreover, we found that students scoring high on impulsive urgency were particularly likely to engage in counterfactual processing at bedtime, suggesting that their rash daytime behavior comes back to haunt them as they try to fall asleep.

Although regrets may occur at all ages, empirical evidence suggests that the experience and effects of this emotion change across the adult life span $[13,14]$. Given that the opportunities to undo the negative consequences of regretted behavior decline with age, it was proposed that in later adulthood, intense regrets might increasingly result in feelings of helplessness and depression, which, in turn, might adversely affect physical health [15]. In light of these hypotheses, we have recently investigated the effect of regrets on sleep in a sample of elderly people, whose age ranged from 51 to 98 years [16]. The pattern of their responses clearly suggested circadian variation in the experience of regrets: While regret frequency remained at comparatively low levels for most of the waking hours, a sharp rise occurred in the evening after going to bed. Furthermore, results revealed that the nocturnal rise of regrets substantially contributed to insomnia severity independently of other wellknown risk factors for late-life sleep disturbance, such as depression, sleep-interfering medical conditions, or medica- 
tions (for these risk factors, see [2]). Finally, as with young adults, rash-action-prone older adults were particularly likely to experience regrets at bedtime and were therefore at a higher risk for sleep disturbances.

Given that regrets may not only contribute to insomnia, but also to a host of other health problems, including persistent skin trouble, recurring stomach trouble, migraine headaches, and depression [15], the questions of how actual regrets can be managed (response-focused emotion regulation) and how potential future regrets may be prevented (antecedent-focused emotion regulation) is of great practical interest (for the distinction between these two types of emotion regulation, see [17]). Although maladaptive management of actual regrets has for some time been recognized as a risk factor for a number of health problems (e.g., $[18,19])$, the role of regret prevention has to date remained largely unexplored in clinically oriented studies.

The lack of clinical investigations into regret prevention is all the more surprising because more than two decades of decision-making research have shown that the anticipation of potential future regrets can exert a strong influence on behavior (for a review, see [20]). The main lesson that emerges from this line of research is that people are motivated to avoid regrets: That is, they basically behave in ways that shield them from experiencing this emotion in the future. However, people may differ in their tendency to preview or prefeel potential future regrets and, as a consequence, in their ability to avoid the experience of actual regrets. More specifically, people who tend to neglect the regrets they might experience in the future may blindly run into situations or adopt behaviors that are prone to evoke regrets. As a consequence, they are likely to experience more actual regrets and related counterfactual emotions. In short, failures in regret anticipation may undermine regret avoidance, thereby increasing regret frequency and ultimately also the risk of regret-related health problems.

The aim of the present study was to test this assumption with regard to insomnia. To assess the tendency to neglect future regrets, we developed a new self-report measure, the Regret Anticipation Failures Scale (RAFS; see below). As detailed previously, we assumed that people who tend to neglect potential future regrets more frequently run into regret-eliciting situations or adopt regret-evoking behaviors and that, as a consequence, they experience more actual regrets and related counterfactual emotions. Furthermore, on the basis of our previous findings $[11,16]$, we expected that these counterfactual emotions would especially emerge at bedtime, thereby interfering with sleep. More specifically, we sought to test the following three hypotheses: (a) Regret anticipation failures are linked to the frequency of regretrelated counterfactual thoughts and emotions at bedtime; (b) regret anticipation failures are linked to insomnia severity; and (c) the frequency of regret-related thoughts and emotions at bedtime mediates, at least partially, the effect of regret anticipation failures on insomnia severity.

\section{MATERIALS AND METHODOLOGY}

\section{Participants and Procedure}

One hundred and five undergraduate students of psychology at the University of Geneva, aged 18 to 45 years $(M=$
22.14; $S D=4.66)$, completed the questionnaires described hereafter. The sample comprised 93 women and 12 men. The students participated to fulfill a course requirement and were assessed in groups of up to 40 people. No screening procedure was used for selecting the participants.

\section{Measures \\ Regret Anticipation Failures Scale (RAFS)}

The RAFS was developed by the first author of this study to evaluate interindividual differences in regret anticipation. In a pilot phase, a series of questions on regret anticipation were repeatedly tested on native French speakers who were invited to comment on the wording of the items. On the basis of the comments and ratings of these participants, seven items were retained for the final version of the RAFS. The instruction of the RAFS reads as follows: "Below you will find several statements on the experience of regrets. Please indicate the degree to which you agree with each statement by circling the appropriate number." The wording of the seven items is as follows: (1) "This is crazy! When I'm relaxed, I can easily anticipate the regrets that certain behaviors could evoke in me. In contrast, when I'm upset, I tend to neglect future regrets that my words or actions could evoke"; (2) "Even when I'm stressed, I can foresee the regrets that certain behaviors could evoke in me"; (3) "If only, in the heat of the action, I managed better to keep in mind that I could one day regret what I'm about to do or to say!"; (4) "It's unbelievable how, even calm, I fail to feel in advance the regrets that my behaviors could entail!"; (5) "I'm astonished at the degree to which, under stress, I'm unaware of the regrets that certain behaviors could evoke in me"; (6) "Sometimes, when I think back on certain behaviors that I later regretted, I'm surprised to have paid so little attention to the regrets that I was bound to feel"; and (7) "In principle, I try to avoid behaviors that I might regret later on, but when I'm stressed, I tend to forget about these future regrets." Answers are given on a 5-point Likert scale ranging from 0 (doesn't apply to me at all) to 4 (fully applies to me). Answers to Item 2 were reverse coded so that higher total scores indicate a more pronounced neglect of future regrets. Analyses revealed that the RAFS captures a single dimension and possesses high internal consistency (see Results).

\section{Bedtime Counterfactual Processing Questionnaire (BCPQ)}

The BCPQ [11] was used to assess the frequency of regret-related counterfactual thoughts and emotions during the presleep period. The questions are preceded by the following instruction: "When in bed in the evening, one sometimes reviews the day that has just come to an end. Hereafter, please indicate how often different kinds of thoughts occur to you as you are trying to get to sleep." In the present study, we used an extended version of the original BCPQ that included three new items (numbered 3, 6, and 9 hereafter) designed to control for response bias effects. These items assess pride-related experiences, thus quite the contrary of feelings of regret, shame, and guilt. The wording of the 10 items is as follows: (1) "After going to bed, how often do you regret your behavior toward others?"; (2) "After going to bed, how often do you think: 'If only I had made another choice!'”; (3) "After going to bed, how often do you feel proud of yourself when you look back at all that you accomplished?"; (4) "After going to bed, how often do you feel 
guilty because you have the impression of having done wrong to others?"; (5) "After going to bed, how often do think: 'If only I were more prudent!"”; (6) "After going to bed, how often do you congratulate yourself for the way you treated other people?"; (7) "After going to bed, how often do you feel ashamed of yourself because of your behavior?"; (8) "After going to bed, how often do you imagine how you would have liked to behave, but did not?"; (9) "After going to bed, how often do you feel happy with the way you handled a conflict with other people?"; and (10) "After going to bed, how often do you feel preoccupied with the consequences of your behavior toward others?" Answers are given on a 5-point Likert scale ranging from 0 (almost never) to 4 (very often). The original BCPQ without the three control items has been shown to capture a single dimension and to possess high internal consistency, with a Cronbach's alpha of .87 .

\section{Insomnia Severity Index (ISI)}

The French version of the ISI [21] was used to evaluate sleep impairment. The ISI contains seven items that are rated on a 5-point Likert scale ranging from 0 (not at all) to 4 (extremely). Respondents are asked to evaluate the following dimensions of insomnia: (a) severity of insomnia during the past month (difficulty falling asleep, difficulty staying asleep, problem waking up too early); (b) satisfaction with current sleep patterns; (c) interference with daytime functioning; (d) noticeability of impairment to significant others; and (e) level of distress caused by the sleep problem. Total scores range from 0 to 28 , with higher scores indicating higher perceived insomnia severity. The French version of the ISI has been shown to possess good internal consistency $(\alpha=.88)$ and to correlate strongly $(r=.67)$ with the Pittsburgh Sleep Quality Index (PSQI; $[21,22])$. Concurrent validity of the ISI has also been demonstrated using otheradministered versions of this instrument, polysomnography, and cardiovascular measures [23, 24]. Given that the ISI does not contain any items on sleep-interfering dreams or medication, two questions of the PSQI were added to the ISI in the present study: Item $5 \mathrm{~h}$ ("During the past month, how often have you had trouble sleeping because you had bad dreams?") and Item 7 ("During the past month, how often have you taken medicine [prescribed or 'over the counter'] to help you sleep?"). Answers to these items are given on a 4point Likert scale ranging from 0 (not during the past month) to 3 (three or more times a week).

\section{RESULTS}

\section{Preliminary Analyses}

Cronbach's alpha coefficients, mean scores, and standard deviations for the three questionnaires are provided in Table 1. The range of alpha coefficients (.75 to .83) suggests that the questionnaires possess sound internal consistency. Given that the RAFS constitutes a novel instrument, we investigated its structure by means of a scree-plot and an exploratory factor analysis using the maximum likelihood estimator. Results indicated that responses to this questionnaire are well reflected by a single-factor solution accounting for $41.2 \%$ of the variance, with factor loadings ranging from .40 (Item 2) to .70 (Item 3).

According to the norms for the ISI [23], 58.1\% of our participants did not show any sign of clinically significant insomnia (score range $=0$ to 7 ), $24.8 \%$ gave evidence of subthreshold insomnia (range $=8$ to 14 ), $16.2 \%$ could be considered as suffering from moderate clinical insomnia (range $=15$ to 21 ), and $0.95 \%$ (corresponding to one participant) obtained a score indicating severe clinical insomnia (range $=22$ to 28 ). For the two PSQI questions, $42.9 \%$ of our participants reported not having had trouble sleeping as a result of bad dreams during the past month, whereas $50.5 \%$ indicated having had such problems less than once a week and $6.7 \%$ once or twice a week. Finally, $7.6 \%$ of our participants reported having taken medicine to help them sleep less than once during the past month and $0.95 \%$ once or twice a week. In the statistical analyses reported below, ISI total scores were entered as a continuous variable, that is: Participants were not divided into the four previously mentioned normative categories.

\section{Correlational Analyses}

To explore the associations between the questionnairebased scores, we computed Pearson correlations. As predicted, failures in regret anticipation were positively related to the frequency of regret-related counterfactual thoughts and emotions at bedtime $(r=.33, P<.001)$, as well as to insomnia severity $(r=.27, P<.01)$. Again as predicted, the frequency of counterfactual processing at bedtime was also positively linked to insomnia severity $(r=.41, P<.001)$.

Interestingly, the frequency of bad, sleep-interfering dreams was positively associated with regret anticipation failures $(r=.29, P<.01)$, counterfactual processing at bedtime ( $r=.20, P<.05)$, and, as might be expected, insomnia severity

Table 1. Cronbach's Alphas, Means, and Standard Deviations for the Questionnaire Scores

\begin{tabular}{|c|c|c|c|}
\hline Variables & $\boldsymbol{\alpha}$ & $\boldsymbol{M}$ & \multicolumn{1}{c|}{ SD } \\
\hline \hline $\begin{array}{c}\text { RAFS } \\
\text { Total score }\end{array}$ & .75 & 8.00 & 4.43 \\
\hline $\begin{array}{c}\text { BCPQ } \\
\text { Total score }\end{array}$ & .81 & 8.64 & 4.44 \\
\hline $\begin{array}{c}\text { ISI } \\
\text { Total score }\end{array}$ & .83 & 7.77 & 5.31 \\
\hline
\end{tabular}

Note. RAFS = Regret Anticipation Failures Scale; BCPQ = Bedtime Counterfactual Processing Questionnaire; ISI = Insomnia Severity Index. 
( $r=.29, P<.01)$. The use of sleeping pills was positively associated with counterfactual processing at bedtime $(r=.20$, $P<.05)$ and insomnia severity $(r=.41, P<.001)$, but unrelated to regret anticipation problems $(r=-.01, P=.89)$.

Finally, to examine the discriminant validity of the $\mathrm{BCPQ}$, we computed the correlations between the three pride-related control items of this scale, regret anticipation failures as assessed by the RAFS, and insomnia severity as evaluated by the ISI. Analyses revealed that the three control items were not significantly related to regret anticipation failures ( $r$ range $=-.011$ to $-.00, P>.28$ ) or to insomnia severity $(r$ range $=-.001$ to $.16, P>.10)$. These results suggest that our main findings cannot be attributed to a simple bias in responding to the $\mathrm{BCPQ}$ or to a generally high emotionality at bedtime.

\section{Regression and Mediation Analyses}

With the aim to explore whether the effect of regret anticipation failures on insomnia severity was mediated by bedtime counterfactual processing, we computed a mediation analysis following the principles set out by Baron and Kenny [25]. Results were as follows: (a) Regret anticipation problems affected frequency of bedtime counterfactual processing $(\beta=.33, t=3.52, P<.001)$; (b) regret anticipation failures affected insomnia severity $(\beta=.27, t=2.83, P<.01)$; (c) frequency of bedtime counterfactual processing affected insomnia severity $(\beta=.36, t=3.78, P<.001)$ when the latter was simultaneously regressed on regret anticipation failures ( $\beta=.15, t=1.63, P=.11)$, the adjusted $R^{2}$ for this model being .17; and (d) according to the Sobel test, the mediator-related reduction in the effect of regret anticipation failures on insomnia severity was significant $(z=2.58, P<.01)$. The mediation analysis thus suggested that frequency of bedtime counterfactual processing mediated the relation between regret anticipation failures and insomnia severity (see Fig. 1).

\section{DISCUSSION}

The results of this study suggest that weak anticipation of potential future regrets leads to a rise of regrets and related counterfactual processing at bedtime, thereby hindering the process of falling asleep. More specifically, our main findings may be summarized as follows: (a) Regret anticipation failures in the course of the day were associated with the frequency of regret-related counterfactual thoughts and emotions at bedtime; (b) regret anticipation failures were also related to insomnia symptoms; and (c) the frequency of regret-related thoughts and emotions at bedtime meditated the effect of regret anticipation failures on insomnia symptoms. Interestingly, failures in regret anticipation and bedtime counterfactual processing were also related to the frequency of bad, sleep-interfering dreams. In line with previous studies $[11,26]$, this association suggests that regret-related counterfactual processing continues during the night and may manifest itself on the mental stage of the dreamer.

To our knowledge, these findings are the first to relate self-assessed difficulties with regret anticipation to a clinically relevant outcome. As reviewed in the introduction, actual regrets have been linked to a host of health problems, including insomnia $[15,16]$, suggesting that problems with the anticipation and prevention of potential future regrets might also have adverse effects on health. The findings of the present study support this assumption by showing that individuals who fail to anticipate future regrets experience more regret-related thoughts and emotions at bedtime and that this surge of counterfactual processing interferes with sleep. Put differently, individuals who successfully anticipate potential future regrets seem to be able to avoid situations or behaviors that are liable to evoke regrets, thereby shielding themselves from sleep-interfering affective arousal. Our findings thus highlight the important implications of antecedent-focused emotion regulation [17] for mental and physical health-currently a largely unexplored field of investigation.

An important issue for future research concerns the exploration of the exact mechanisms whereby potential future regrets may be anticipated and effectively avoided. In this context, a finding from two of our previous investigations is of particular relevance: In contrast to urgency, the facet of impulsivity termed lack of premeditation was unrelated to the experience of counterfactual emotions at bedtime [11, 16]. Premeditation refers to the tendency to think and reflect on the consequences of an act before engaging in that act [27]. Lack of premeditation thus denotes deficiencies in a cognitive, "cool" type of anticipation, whereas the items of the RAFS are geared toward difficulties in an affective, "hot" type of anticipation. In accord with this distinction, accumulating evidence suggests that advantageous decision making is associated with two distinct systems: an explicit system that conveys conscious knowledge, and an implicit system that is based on emotion-related signals, also known

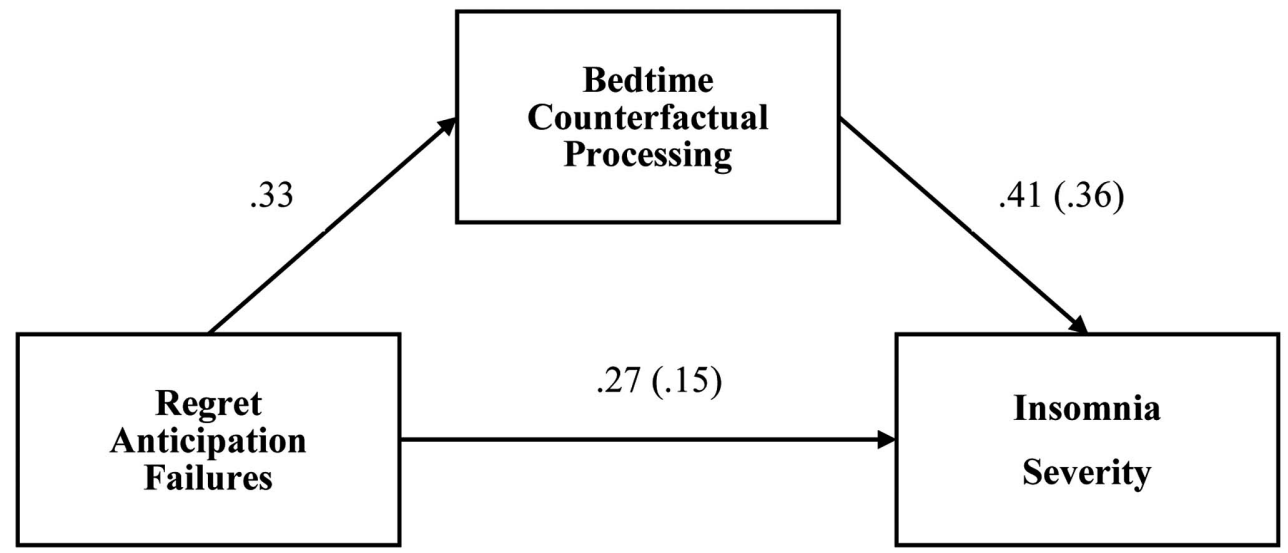

Fig. (1). Mediation analysis for the effect of regret anticipation failures on insomnia severity. Standardized coefficients are given for the simple regression analyses and the multiple regression analysis (in parentheses). 
as somatic markers [28]. Taken together, the results of our two previous investigations $[11,16]$ and of the present study thus suggest that only hot regret anticipation failures entail a rebound of regrets at bedtime, thereby interfering with the process of falling asleep.

Our findings pertaining to failures in hot regret anticipation also raise the question of the processes that specifically impede this type of affective forecasting. An intriguing explanatory perspective emerges if we assume an interplay between the management of actual regrets and that of potential future regrets. Individuals who tend to neglect future regrets likely end up experiencing more actual regrets, as the results of our study suggest. Given that actual regrets are negatively valenced emotions, people may be motivated to mentally avoid them, for example, by engaging in suppression [12], as the elderly participants of our previous study indicated doing [16]. However, given that suppression supposes attentional control processes and that the latter are attenuated by the process of falling asleep, the use of this strategy may result in a rebound of regrets at bedtime [29]. Moreover, habitual suppression of actual regrets may also dampen the experiential simulation of future regrets. In sum, a vicious cycle may develop: People who fail to anticipate future regrets will incur more actual regrets, and suppression of the latter will blind them still further to future regrets.

In addition to suppression, interference from competing emotional experiences-for instance, a fit of anger-may momentarily override the awareness of potential future regrets. More research is needed to address these alternative but not mutually exclusive accounts of blurred regret anticipation. A promising avenue for clinical investigations will be to explore the boundaries of adaptive regret anticipation. In this context, it is noteworthy that not only underestimation, but also overestimation of future regrets [30] may contribute to clinically relevant problems, for instance, procrastination [31]. Ironically, this form of avoidance of regrets of action typically leads to regrets of inaction [12]. Thus, a challenge for clinically oriented studies will be to determine how people can successfully navigate between the under- and overestimation of future regrets.

Regarding insomnia in particular, the question arises as to how our findings on affective processes may feed into current cognitive models of insomnia. As reviewed earlier, a central tenet of these models is that excessive mental activity at bedtime may be considered as the main impediment to sleep [5, 6]. Researchers have also proposed that cognitive arousal at bedtime can take the form of worry or rumination and that the two may be distinguished temporally: Worry refers to distress regarding future events, whereas rumination concerns thoughts of past events and current symptoms (e.g., [32]). In light of this distinction, future research will need to determine whether rumination (repetitive thoughts of past events) and regret (negative emotions connected to thoughts about how different past behaviors might have yielded better outcomes) essentially overlap or whether they independently relate to insomnia.

To overcome the limitations of a cross-sectional design such as ours, which does not allow causal inferences, future studies might adopt longitudinal or experimental designs to investigate the cognitive-affective interplay of sleepinterfering mentation. Along these lines, we have recently conducted a study with young adults revealing that the experimental induction of regrets before bedtime may delay sleep onset, thus corroborating a causal link between nocturnal regrets and insomnia [33]. An open question that awaits further investigation is that of whether regrets might have a stronger impact on sleep in women than in men, given that women have been found to be comparatively more prone to experience counterfactual emotions (e.g., [34]). In view of the predominantly female composition of the sample, this question could not be addressed in the present study. Another limitation of this study results from the fact that no information was collected on mental health or other factors that might be associated with insomnia, such as medications or alcohol and caffeine consumption.

Of note for future investigations, Roese and colleagues [19] recently found that both repetitive thought and regret were associated with general distress, whereas only regret was linked to anhedonic depression and anxious arousal. These findings are suggestive of the ways in which affective approaches may complement cognitive conceptualizations of insomnia: If affective factors contribute to insomnia over and above the influence of well-established cognitive factors, their identification will not only expand our theoretical knowledge, but also spur the development of new intervention and prevention techniques. For example, new techniques might foster affect anticipation to avoid a buildup of negative emotions in the course of the day or they might promote positive emotions such as gratitude at the end of the day [10].

\section{ACKNOWLEDGEMENTS}

This research was supported by the National Center of Competence in Research (NCCR) in Affective Sciences financed by the Swiss National Science Foundation (51NF40104897) and hosted by the University of Geneva.

\section{Appendix: Cognitive and Affective Terminology}

\begin{tabular}{|l|l|}
\hline Term & Definition \\
\hline \hline Counterfactual thoughts & $\begin{array}{l}\text { Mental representations of alternatives to past events, actions, or states [35-37]. Example: "If only I had studied more, I } \\
\text { would have passed the exam." }\end{array}$ \\
\hline Counterfactual emotions & $\begin{array}{l}\text { Affective reactions (e.g., regret, shame, guilt) resulting from the counterfactual comparison of reality with what might } \\
\text { have been [12, 35]. }\end{array}$ \\
\hline Regret & $\begin{array}{l}\text { Emotion experienced when realizing that the current situation would be better if only one had acted differently in the } \\
\text { past [12]. }\end{array}$ \\
\hline Repetitive thought & $\begin{array}{l}\text { Process of thinking attentively, repetitively, or frequently about oneself and one's world. Rumination and worry may } \\
\text { be considered as subtypes of repetitive thought [38-40]. }\end{array}$ \\
\hline Rumination & Subtype of repetitive thought, mainly concerning past events [32, 38]. \\
\hline
\end{tabular}




\begin{tabular}{|l|l|}
\hline Worry & Subtype of repetitive thought, mainly concerning potentially threatening future events [32, 38]. \\
\hline $\begin{array}{l}\text { Antecedent-focused emotion } \\
\text { regulation }\end{array}$ & $\begin{array}{l}\text { Emotion regulation processes that occur before appraisals give rise to full-blown emotional response tendencies [17]. } \\
\text { Example: anticipation and avoidance of future regrets. }\end{array}$ \\
\hline $\begin{array}{l}\text { Response-focused emotion } \\
\text { regulation }\end{array}$ & $\begin{array}{l}\text { Emotion regulation processes that occur after an emotional response has been generated [17]. Example: suppression of } \\
\text { actual regrets. }\end{array}$ \\
\hline
\end{tabular}

\section{REFERENCES}

[1] National Sleep Foundation. 2009 sleep in America poll. Washington, DC: National Sleep Foundation.

[2] Mai E, Buysse DJ. Insomnia: prevalence, impact, pathogenesis, differential diagnosis, and evaluation. Sleep Med Clin 2008; 3: 167-74.

[3] Harvey AG, Tang NKY, Browning L. Cognitive approaches to insomnia. Clin Psychol Rev 2005; 25: 593-611.

[4] Morin CM, Bootzin RR, Buysse DJ, Edinger JD, Espie CA, Lichstein KL. Psychological and behavioral treatment of insomnia: update of the recent evidence (1998-2004). Sleep 2006; 29: 13981414.

[5] Harvey AG. A cognitive model of insomnia. Behav Res Ther 2002; 40: 869-93.

[6] Espie CA, Broomfield NM, MacMahon KMA, Macphee LM, Taylor LM. The attention-intention-effort pathway in the development of psychophysiologic insomnia: a theoretical review. Sleep Med Rev 2006; 10: 215-45.

[7] Walker MP, Harvey AG. Obligate symbiosis: sleep and affect. Sleep Med Rev 2010; 14: 215-17.

[8] Baglioni C, Spiegelhalder K, Lombardo C, Riemann D. Sleep and emotions: a focus on insomnia. Sleep Med Rev 2010; 14: 227-38.

[9] McCullough ME, Emmons RA, Tsang J-A. The grateful disposition: a conceptual and empirical topography. J Pers Soc Psychol 2002; 82: 112-27.

[10] Wood AM, Joseph S, Lloyd J, Atkins S. Gratitude influences sleep through the mechanism of pre-sleep cognitions. J Psychosom Res 2009; 66: 43-48.

[11] Schmidt RE, Van der Linden M. The aftermath of rash action: sleep-interfering counterfactual thoughts and emotions. Emotion 2009; 9: 549-53.

[12] Zeelenberg M, Pieters R. A theory of regret regulation 1.0. J Consum Psychol 2007; 17: 3-18.

[13] Lecci L, Okun M A, Karoly P. Life regrets and current goals as predictors of psychological adjustment. J Pers Soc Psychol 1994; 66: 731-41.

[14] Wrosch C, Heckhausen J. Perceived control of life regrets: good for young and bad for old adults. Psychol Aging, 2002; 17: 340-50.

[15] Wrosch C, Bauer I, Scheier MF. Regret and quality of life across the adult life span: the influence of disengagement and available future goals. Psychol Aging 2005; 20: 657-70.

[16] Schmidt RE, Renaud O, Van der Linden M. Nocturnal regrets and insomnia in elderly people. Manuscript submitted for publication.

[17] Gross JJ, Thompson RA. Emotion regulation: conceptual foundations. In: Gross, JJ, ed. Handbook of emotion regulation. New York: Guilford Press 2007: 3-24.

[18] Heckhausen J, Wrosch C, Schulz, R. A motivational theory of lifespan development. Psychol Rev 2010; 117: 32-60.

[19] Roese NJ, Epstude K, Fessel F, et al. Repetitive regret, depression, and anxiety: findings from a nationally representative survey. J Soc Clin Psychol 2009; 28: 671-88.

[20] Zeelenberg M., Pieters R. On the consequences of mentally simulating future forgone outcomes: a regret regulation perspective. In: Markman KD, Klein WMP, Suhr JA, eds. Handbook of imagina- tion and mental simulation New York: Psychology Press 2009: 413-24.

[21] Blais FC, Gendron L, Mimeault V, Morin CM. Evaluation de l'insomnie: validation de trois questionnaires [Evaluation of insomnia: validation of three questionnaires]. Encephale 1997; 23: 447-53.

[22] Buysse DJ, Reynolds CF III, Monk TH, Berman SR, Kupfer DJ. The Pittsburgh Sleep Quality Index: a new instrument for psychiatric practice and research. Psychiatry Res 1989; 28: 193-213.

[23] Bastien CH, Vallières A, Morin CM. Validation of the Insomnia Severity Index as an outcome measure for insomnia research. Sleep Med 2001; 2: 297-307.

[24] Schmidt RE, Richter M, Gendolla GHE, Van der Linden M. Young poor sleepers mobilize extra effort in an easy memory task: evidence from cardiovascular measures. J Sleep Res 2010; 19: 487-95.

[25] Baron RM, Kenny DA. The moderator-mediator variable distinction in social psychological research: conceptual, strategic, and statistical considerations. J Pers Soc Psychol 1986; 51: 1173-82.

[26] McNamara P, Andresen J, Arrowood J, Messer G. Counterfactual cognitive operations in dreams. Dreaming 2002; 12: 121-33.

[27] Whiteside SP, Lynam DR. The five factor model and impulsivity: using a structural model of personality to understand impulsivity. Pers Indiv Differ 2001; 30: 669-89.

[28] Guillaume S, Jollant F, Jaussent I, Lawrence N, Malafosse A, Courtet P. Somatic markers and explicit knowledge are both involved in decision-making. Neuropsychologia 2009; 47: 2120-24.

[29] Schmidt RE, Gendolla GHE. Dreaming of white bears: the return of the suppressed at sleep onset. Conscious Cogn 2008; 17: 714-24.

[30] Gilbert DT, Morewedge CK, Risen JL, Wilson TD. Looking forward to looking backward: the misprediction of regret. Psychol Sci 2004; 15: 346-350.

[31] Anderson CJ. The psychology of doing nothing: forms of decision avoidance result from reason and emotion. Psychol Bull 2003; 129: 139-167.

[32] Kaplan KA, Talbot LS, Harvey AG. Cognitive mechanisms in chronic insomnia: processes and prospects. Sleep Med Clin 2009; 4: 541-548.

[33] Schmidt RE, Van der Linden M. Feeling too regretful to fall asleep: an experiment on affective factors in insomnia. Manuscript submitted for publication.

[34] Tangney JP, Dearing RL. Shame and guilt. Guilford Press: New York, 2002.

[35] Kahneman D, Miller DT. Norm theory: comparing reality to its alternatives. Psychol Rev 1986; 93: 136-153.

[36] Byrne RMJ. Mental models and counterfactual thoughts about what might have been. Trends Cogn Sci 2002; 6: 426-431.

[37] Epstude K, Roese NJ. The functional theory of counterfactual thinking. Pers Soc Psychol Rev 2008; 12: 168-192.

[38] Segerstrom SC, Tsao JCI, Alden LE, Craske MG. Worry and rumination: repetitive thought as a concomitant and predictor of negative mood. Cogn Ther Res 2000; 24: 671-688.

[39] Segerstrom SC, Stanton AL, Alden LE, Shortridge BE. A multidimensional structure for repetitive thought: what's on your mind, and how, and how much? J Pers Soc Psychol 2003; 85: 909-921.

[40] Watkins ER. Constructive and unconstructive repetitive thought. Psychol Bull 2008; 134: 163-206.

Received: February 25, 2011

Revised: April 06, 2011

Accepted: April 08, 2011

(C) Schmidt and Van der Linden; Licensee Bentham Open.

This is an open access article licensed under the terms of the Creative Commons Attribution Non-Commercial License (http://creativecommons.org/licenses/by-nc/3.0/) which permits unrestricted, non-commercial use, distribution and reproduction in any medium, provided the work is properly cited. 